\title{
Zwischen Erbleiden und Infektionskrankheit: Wahrnehmung und Umgang mit Tuberkulose im Nationalsozialismus
}

\author{
Between Hereditary Ailment and Infectious Disease: the Perception \\ and Treatment of Tuberculosis under National Socialism
}

„Tb ist auch eine politische Seuche“ - so titelte eine große bayerische Regionalzeitung anlässlich des Welttuberkulosetags im März 2005. Denn für die derzeitige starke Zunahme der Erkrankungsfälle seien vor allem politische Faktoren verantwortlich, wie die ,riesige Armut“ in der Dritten Welt, „gepaart mit chronischer Unterernährung, sozialer Verelendung in den Slums wachsender Millionenstädte“ sowie „Bürgerkriege mit Vertreibung der Bevölkerung“ [1]. Diese Wahrnehmung der Tuberkulose als eine auch „politische“ Angelegenheit ist nicht neu: Bereits in früheren Jahrhunderten wurde ein Zusammenhang zwischen „Krankheit und sozialer Lage“ - so der Titel eines sozialmedizinischen Klassikers aus dem Jahr 1913 [2] - hergestellt und auch im Nationalsozialismus wurde der Tuberkulose unter anderem mit politischen Maßnahmen zu begegnen versucht. Im Unterschied zur Zeit vor 1933 zielten diese Maßnahmen aber nicht mehr auf eine Verbesserung der Lebensbedingungen Erkrankter und Gefährdeter ab, sondern auf die soziale Ausgrenzung Tuberkulöser zum „Schutze des Volkskörpers“. Denn Tuberkulose galt im „Dritten Reich“ als eine „asoziale Krankheit“.

\section{Vom romantischen Künstlerleiden zur asozialen Krankheit}

Die kollektive Wahrnehmung der Tuberkulose, die stets auch den gesellschaftlichen Umgang mit ihr beeinflusste, hat sich in der Neuzeit mehrfach gewandelt [3]. Im 18. Jahrhundert, als das Leiden noch selten war, entstand das schwärmerische Bild einer „romantischen Krankheit“. Anfällig für Schwindsucht - so das von zeitgenössischen Schriftstellern, Opernlibrettisten, Malern und vereinzelt auch von Medizinern vermittelte Bild - waren besonders sensible und äußerst leidenschaftliche Individuen, also vor allem Künstler und Intellektuelle. Diese verklärte Vorstellung wich zur Mitte des 19. Jahrhunderts einer anderen Wahrnehmung der Krankheit. Bedingt durch ein epidemisches Auftreten der Tuberkulose setzte sich seit etwa 1850 allmählich die Sichtweise durch, dass es sich um eine „proletarischen Krankheit“ handele [4]. Als Krankheitsursache wurde neben konstitutioneller Disposition - und, wie seit 1882 bekannt war, einer Infektion mit dem spezifischen Erreger - in erster Linie die mangelhafte Arbeits-, Wohn- und Ernährungssituation der Unterschichten angesehen. Zur Bekämpfung der „Volksseuche“ verlangten die Vertreter der jungen Spezialdisziplin der Sozialhygiene daher vor allem wohlfahrtsstaatliche Reformen und sozialmedizinische Präventionsmaßnahmen.

In der Zeit des Nationalsozialismus wurde das Leiden dann von einer Krankheit der Armen zu einem Kennzeichen von Asozialität umgedeutet. Vor dem Hintergrund der von NS-Politikern proklamierten „Pflicht zur Gesundheit“ kam Krankheit grundsätzlich einem „Pflichtversäumnis“ und „Versagen“ gleich [5]. Tuberkulöse, die als „unheilbar“ und „uneinsichtig“ galten, wurden zu „asozialen Bazillenstreuern“ herabgewürdigt, gegen die „mit Zwangsmaßnahmen“ vorzugehen war [6]. Da sie nach NS-Anschauung für die „Volksgemeinschaft“ wertlos waren, wurden sie sozial vernachlässigt, zum Teil systematisch unterversorgt und in Konzentrationslagern sogar gezielt getötet.

Rubrikherausgeber: R. Kropp (federführend), U. Costabel, H. S. Fuchs, C. Habrich, H. Jungbluth, H. J. Klippe, N. Konietzko, R. Loddenkemper, G. Neumann, S. Schulz, M. Teschner 


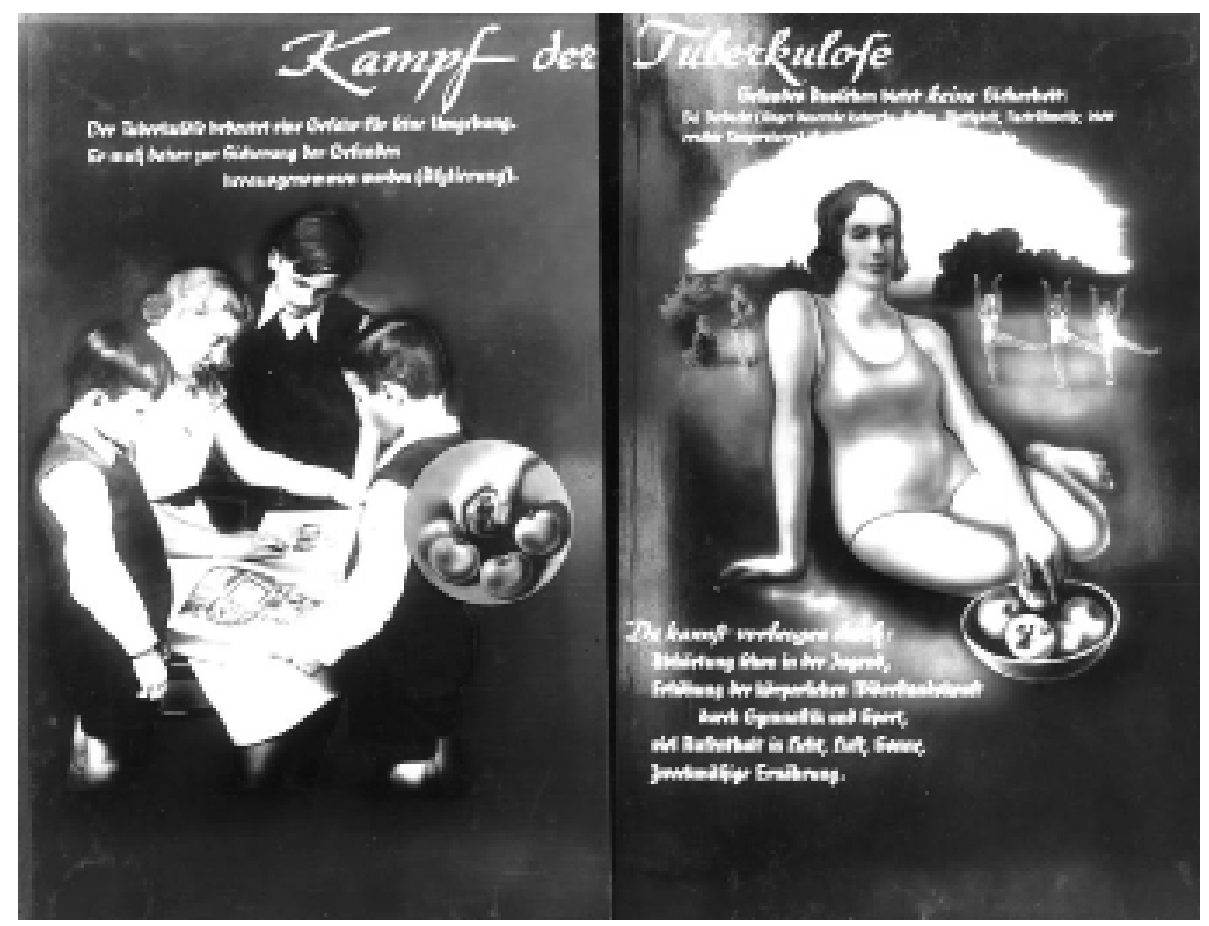

Abb. 1 „Kampf der Tuberkulose“. Tafel einer Wanderausstellung des Deutschen Hygienemuseums, Dresden, ca. 1939, Stiftung Deutsches Hygienemuseum.

Infektion oder Erbleiden? Theorien zur Ätiologie

Mit der Entdeckung des Mycobacterium tuberculosis im Jahr 1882 hatte Robert Koch (1843-1910) zwar die Richtigkeit der Ansteckungstheorie bei Tuberkulose bewiesen. Die seit der Antike nachweisbare Diskussion allerdings, ob es sich bei dem Leiden um eine Infektions- oder um eine Erbkrankheit handele [7], hatte er damit jedoch nicht dauerhaft beendet. Auch deutete ein Rückgang der Tuberkulosesterblichkeit von Arbeitern infolge verbesserter Lebensbedingungen, der im späten 19. Jahrhundert zu beobachten war, auf den Einfluss weiterer Faktoren jenseits von Ansteckung und Disposition zur Krankheit hin. Seit Beginn des 20. Jahrhunderts führten daher sogar Repräsentanten der eigentlich auf die gesellschaftliche Bedingtheit von Krankheit fokussierten Sozialhygiene die Tuberkulose auf ein Ursachengeflecht aus Infektion, Konstitution und Milieu zurück. Alfred Grotjahn (1869 - 1931), einer der wichtigsten Fachvertreter in spätem Kaiserreich und Weimarer Republik, plädierte schon vor dem Ersten Weltkrieg für sozialprophylaktische und für eugenische Maßnahmen [8].

Nach dem Machtantritt der Nationalsozialisten nahm die öffentliche Bedeutung der Vererbungsforschung - und in der Folge auch die Summe der dafür bereitgestellten staatlichen Fördergelder - stark zu. Vor diesem Hintergrund wuchs auch das Interesse an der Rolle des Erbfaktors bei der Entstehung der Tuberkulose beträchtlich. Als Beweis für die Heredität des Leidens galten die Zwillingsforschungen des Tuberkulosearztes Karl Diehl (1896-1969) und des Anthropologen Otmar von Verschuer (1896 - 1969) [9]. Die beiden Wissenschaftler hatten eine familiäre Häufung im Auftreten der Erkrankung beobachtet, welche sie als Hinweis auf die Existenz einer genetischen Veranlagung zur Krankheit interpretierten: Eine „hohe Bedeutung des Erbes bei der Entstehung und dem Ablauf der Tuberkulose“ hielten sie deshalb für „erwiesen“ [10]. Da Tuberkulose dennoch ganz unbestreitbar eine exogene Infektionskrankheit war, wurde sie in der NS-Zeit als Mittelding zwischen einer Erbkrankheit und einem Infektionsleiden betrachtet. Diese Zwischenstellung zwischen Erb- und Infektionskrankheit spiegelte auch das AntiTuberkulose-Programm der Nationalsozialisten wider, das eugenische und seuchenprophylaktische Maßnahmen miteinander kombinierte. (Abb.1).

\section{Verhütung von Nachwuchs: Eugenische Maßnahmen}

Obwohl es nicht an diesbezüglichen Forderungen aus der Ärzteschaft fehlte, hatte die NS-Regierung die Tuberkulose nicht als Indikation zur eugenischen Sterilisierung in das „Gesetz zur Verhütung erbkranken Nachwuchses“ von 1933 aufgenommen, weil die exogene Natur der Krankheit nur allzu offensichtlich war [11]. Anders als psychisch Kranke sowie geistig oder körperlich Behinderte konnten somit Tbc-Patienten nicht gegen ihren Willen unfruchtbar gemacht werden. Tuberkulose stellte auch keine gesetzlich sanktionierte Indikation für einen eugenischen Schwangerschaftsabbruch dar [12].

Es wurde in der NS-Zeit jedoch auf andere Weise versucht, die zu „Trägern minderwertigen Erbguts“ herabgewürdigten Tuberkulösen an der Fortpflanzung zu hindern. So konnte diesen Menschen etwa das 1933 eingeführte „Ehestandsdarlehen“ verweigert werden, ein einmaliger zinsloser Kredit von durchaus beträchtlichem Umfang, den der NS-Staat „arisch-erbgesunden“ Brautpaaren gewährte. Erklärtes Ziel des Darlehens war eine Hebung der Geburtenrate, denn für jedes der Ehe entstammende Kind wurde den Darlehensnehmern ein Viertel der Tilgungssumme erlassen. Wie der Gesetzgeber festgelegt hatte, zählten TbcKranke nicht zu den als kreditwürdig erachteten Brautleuten [13]. Ab 1935 gab es zudem die Möglichkeit, Tuberkulösen sogar das Heiraten zu verbieten: Grundlage war das „Gesetz zum Schutze der Erbgesundheit des deutschen Volkes“, mit dem die NS-Regierung Eheverbote bei einer Reihe „eugenisch uner- 


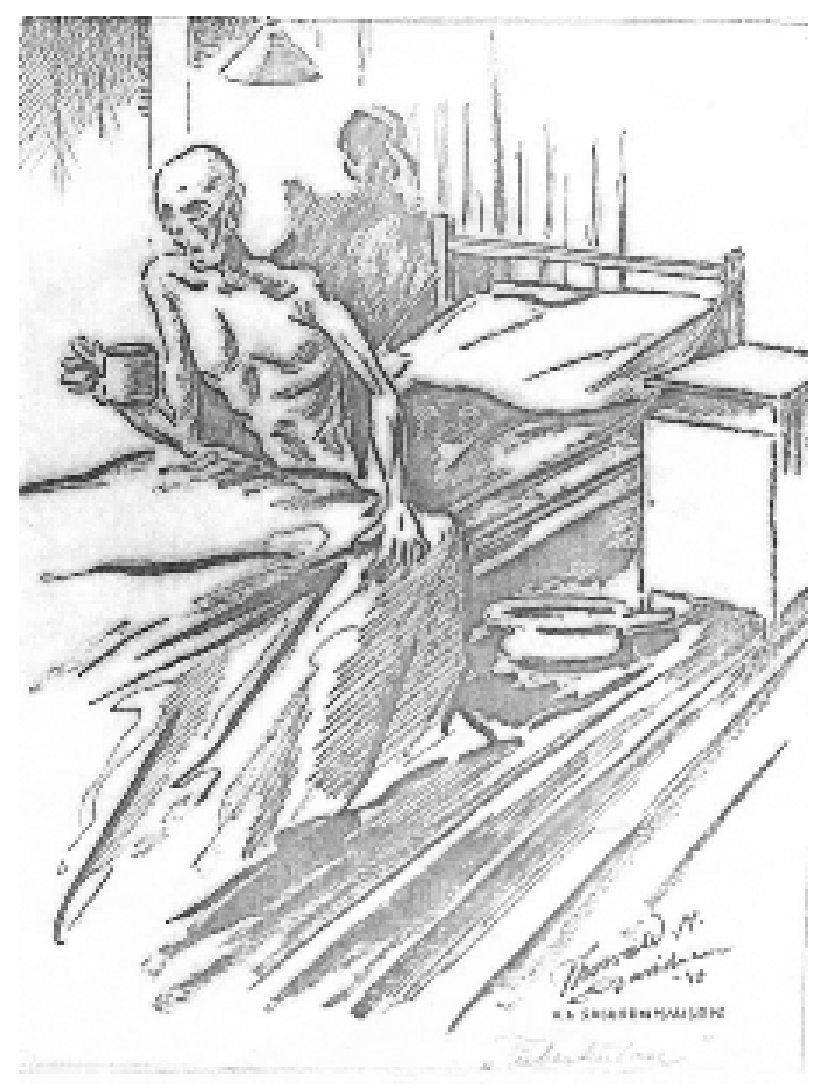

Abb. 2 „Tuberkulose“. Zeichnung von Thorwald Davidsen, Häftling im KZ Sachsenhausen, 1943, Gedenkstätte und Museum Sachsenhausen.

wünschter“ Krankheiten verhängte - darunter auch Lungentuberkulose [14]. Über diese Maßnahmen zur Erschwerung bzw. Verhinderung von Eheschließungen Tuberkulöser hinaus führte die NS-Regierung 1938 eine Regelung ein, die es gesunden Personen erleichterte, sich von einem Tbc-kranken Ehepartner scheiden zu lassen. Wie die anderen genannten Maßnahmen auch sollte die Regelung in erster Linie bei schweren oder chronisch offenen Lungentuberkulosen zur Anwendung kommen [15].

\section{Zwangsisolierung „asozialer Bazillenstreuer“}

Tbc-Kranke galten in der NS-Zeit aber nicht nur als „Gefahr für die deutsche Erbgesundheit“" sondern auch als Ansteckungsquelle und möglicher „Seuchenherd“. Mit der im Dezember 1938 erlassenen „Verordnung zur Bekämpfung übertragbarer Krankheiten“ [16] hob der Gesetzgeber daher bei Tuberkulose das ärztliche Verschwiegenheitsgebot auf und statuierte eine Meldepflicht innerhalb von 24 Stunden. Zur Anzeige verpflichtet waren neben Ärzten und Pflegepersonal auch Privatleute aus dem Umfeld der Erkrankten. Adressat solcher Meldungen war das staatliche Gesundheitsamt, das umgehend die örtliche Polizei verständigte und mit Ermittlungen über „Ursache, Art, Ansteckungsquelle und Ausbreitung der Krankheit“ begann [17]. Sah sich der Amtsarzt dabei von „Uneinsichtigkeit sowie offenem oder verstecktem Widerstreben der Kranken“ behindert, konnte Zwangsasylierung angeordnet werden, auch wenn in der Realität „die Kostenfrage oft die notwendige langdauernde Aussonderung er- schwert[e]“, wie ein Amtsarzt aus dem Münsterland beklagte [18].

Staatlichen Vorgaben zufolge waren vor allem ansteckungsfähige Lungen- oder Kehlkopftuberkulöse zu isolieren, die sich (amts-)ärztlichen Anordnungen gegenüber uneinsichtig zeigten. Für die Zwangsasylierung kamen neben Krankenhäusern vor allem spezielle Anstalten infrage, wie sie z.B. in Thüringen, Brandenburg und Westfalen entstanden. Die älteste und bekannteste derartige Einrichtung, die „Abteilung für zwangsweise Absonderung Offentuberkulöser" der psychiatrischen Heilanstalt Stadtroda (Thüringen), war bereits 1934 auf der Basis der dortigen Landesseuchenordnung eröffnet worden. Hier wurden polizeilich eingewiesene „asoziale oder unbelehrbare Kranke“ unter gefängnisähnlichen Bedingungen und bei knapper Verpflegung „zwangsverwahrt“ [19]. War es in Stadtroda anfangs vor allem um eine Absonderung Infektiöser gegangen, so führte man dort seit 1939 - zumindest bei manchen Insassen - auch therapeutische Maßnahmen durch. Über die Auswahl der Therapierten gibt die Publikation eines Stadtrodaer Abteilungsarztes Auskunft. Danach wurden nur solche Personen in die Behandlung einbezogen, „deren Krankheitsbefund Aussicht auf Entseuchung bot und bei denen sich aufgrund ihres sozialen Wertes therapeutische Maßnahmen lohnten“. Die als „sozial wertlos“ klassifizierten, übrigen Insassen hingegen wurden nicht therapiert. Für sie wurde „überhaupt nichts getan, was den schicksalsmäßigen Ablauf ihres Lungenleidens entscheidend beeinflussen und ihr für die Volksgemeinschaft offenbar unnützes Leben verlängern könnte“, wie auch Anstaltsleiter Gerhard Kloos (1906-1988) in bemerkenswerter Offenheit berichtete [20]. Die in Stadtroda praktizierte Aussonderung und gezielte Vernachlässigung als „lebensunwert" eingestufter Tuberkulöser entsprach der in nationalsozialistischen Konzentrations- und Vernichtungslagern üblichen Selektion.

\section{Das Schicksal Tbc-kranker KZ-Häftlinge}

In Konzentrationslagern trat Tuberkulose so massenhaft auf, dass man fast schon von einem „typischen KZ-Phänomen“ sprechen kann. Konzentrationslager waren der SS unterstellt und damit der ordentlichen Rechtsprechung entzogen. Zentraler Bestandteil des dort herrschenden Terrors waren die unmenschlichen und auf Dauer tödlichen Lebensbedingungen für die Häftlinge, die in primitiven Holzbaracken unter völlig unzureichenden hygienischen Bedingungen zusammengepfercht und - spätestens seit Kriegsbeginn - systematisch mangelernährt wurden (Abb.2). Solche Verhältnisse waren ein geeigneter Nährboden für Tuberkulose, wie der ehemalige Häftling des KZ Sachsenhausen, Günther Wackernagel (1916-2001), berichtete: „Von der Tbc, die sich langsam als eine Seuche innerhalb des Lagers ausbreitete, wurde kein Organ oder Organsystem der Erkrankten verschont. Von allen Tbc-Arten war die Lungentuberkulose die schwerste und weit verbreitete Krankheit unter den Häftlingen. Sie wurde durch die Lebensbedingungen im Lager, das sehr enge Beisammensein der Häftlinge am Tage auf dem Arbeitsplatz und in der Nacht auf dem Strohlager, Mann neben Mann, verbreitet. Durch Husten und Sputumauswurf eines Erkrankten wurde diese Krankheit auf andere Häftlinge übertragen “ [21]. 

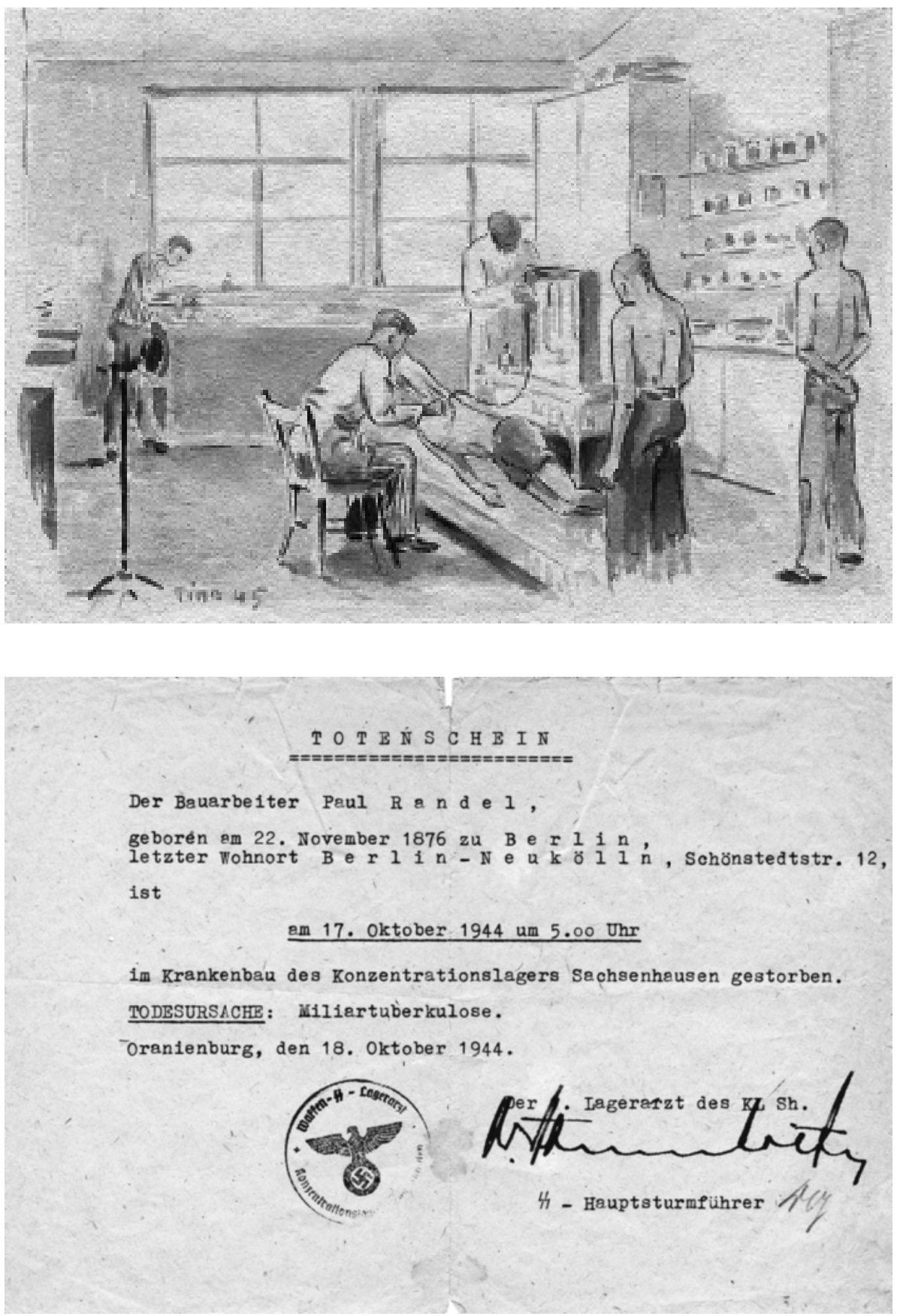

Abb. 3 Gasbrustbehandlung im Krankenrevier. Aquarell von Walter Timm, Häftling des KZ Sachsenhausen, 1945, Gedenkstätte und Museum Sachsenhausen.
Abb. 4 „Todesursache: Militartuberkulose“. Totenschein für den Häftling Paul Randel, unterzeichnet von SS-Lagerarzt Dr. Heinz Baumkötter, 18.10.1944, Gedenkstätte und Museum Sachsenhausen.

\section{Aussonderung und Tod}

Das Vorgehen gegenüber tuberkulösen KZ-Insassen reichte von der Versorgung bis zur „Vernichtung“. Nicht selten erhielten TbcKranke zunächst eine - wenn auch minimale - medizinische Versorgung im Häftlingskrankenbau. Im Krankenrevier des KZ Sachsenhausen gab es schon früh eine eigene Tbc-Abteilung, die aus zwei Krankensälen, Sanitärräumen und einem Zimmer mit einem Pneumothorax-Gerät bestand (Abb.3). Doch eine Gasbrustbehandlung dürften - vor allem in Zeiten großer Verbreitung der Krankheit - nur wenige tuberkulöse Häftlinge erhalten haben. In den meisten Fällen beschränkte sich die Therapie wohl auf Bettruhe und gelegentliche Medikamentengaben zur Linderung der Symptome. Im Jahr 1944 hatte die Tuberkulose in Sachsenhausen ein solches Ausmaß erreicht, dass die Tbc-Abteilung eine ganze Baracke einnahm (Abb.4). Die durchschnittliche Be- legungsstärke betrug 1944 zwischen 800 und 900 Patienten. Wie der als Häftlingsarzt eingesetzte, inhaftierte Mediziner Leo Clasen (geb. 1909) später vor Gericht bezeugte, führte ein SS-Lagerarzt von Zeit zu Zeit Selektionen in der Tbc-Abteilung durch. Die dabei ausgesonderten Kranken „ohne Heilungsaussicht“ wurden mit so genannten „Krankentransporten“ in andere Lager abgeschoben, gezielt vernachlässigt oder durch Giftinjektionen ermordet [22].

Die Furcht vor solchen Selektionen hielt viele erkrankte Häftlinge davon ab, sich im Krankenrevier behandeln zu lassen. Damit entrannen sie aber nicht der Gefahr, als „unnütze Esser“ ausgesondert zu werden, wenn sie aufgrund ihrer Erkrankung die geforderten Arbeitsleistungen nicht mehr erbringen konnten. $\mathrm{Ab}$ Mitte 1940 transportierten viele Konzentrationslager ihre geschwächten und nicht mehr voll arbeitsfähigen Häftlinge in un- 
regelmäßigen Abständen nach Dachau, wo sie - im Lager kursierenden Gerüchten zufolge - im KZ-eigenen Heilkräutergarten leichtere Arbeit bekommen sollten. Seit Ende 1943 gingen diese „Invalidentransporte“ dann in das Konzentrationslager LublinMajdanek und ins KZ Bergen-Belsen, das ab Frühjahr 1944 als „Sterbelager“ diente. Unter den Opfern dieser Transporte, die für die große Mehrheit der Betroffenen den sicheren Tod bedeuteten, waren auch viele Tuberkulöse. Für das KZ Sachsenhausen sind sogar mehrere Transporte bezeugt, die ausschließlich aus Tbc-Kranken bestanden. Bei den Selektionen nutzten die dortigen SS-Ärzte auch das lagereigene Röntgengerät: So wurden z.B. im Sommer 1942 und im Herbst 1944 jeweils Reihenuntersuchungen der gesamten Lagerpopulation vorgenommen und in der Folge auch viele zuvor nicht als krank erkannte Häftlinge „auf Invalidentransport" geschickt [23]. Wie viele andere Selektierte wurden diese Häftlinge einzig wegen einer Tbc-Erkrankung ausgesondert und ermordet.

\section{Missbrauch für medizinische Experimente}

Tuberkulöse wurden außerdem als Versuchspersonen bei medizinischen Experimenten missbraucht. In fast allen Konzentrationslagern fanden seit Kriegsbeginn Menschenversuche an Häftlingen statt. Die Experimente wurden teilweise im Auftrag der SS oder bestimmter Wehrmachtsteile vorgenommen, teilweise ergriffen Wissenschaftler aus zivilen Forschungseinrichtungen selbst die Initiative, um etwa neue Impfstoffe an KZ-Häftlingen testen zu können. Manche Experimente dienten der Klärung von als „kriegswichtig“ angesehenen Fragen, andere waren als Bausteine für wissenschaftliche Karrieren gedacht. Diese Versuche an KZ-Häftlingen waren medizinische Verbrechen, denn die Ärzte experimentierten dabei an Menschen, die nicht frei über eine Versuchsteilnahme entscheiden konnten. Außerdem nahmen die Experimentatoren dabei eine körperliche Schädigung oder auch den Tod - ihrer Probanden in Kauf. In einigen Fällen war der Tod der Versuchsperson sogar geplanter Teil des Versuchs [24].

Bei den Experimenten an Tuberkulösen ging es offenbar zumeist um die Erprobung neuer Heilverfahren. Für den Zeitraum von 1941 - 1945 konnten bisher vier großangelegte Tuberkuloseversuche nachgewiesen werden: Die Experimente des SS-Lagerarztes Waldemar Hoven (1903-1948) im KZ Buchenwald [25], die eines Heilpraktikers und mehrerer SS-Ärzte in Dachau [26], die Versuche von Kurt Heißmeyer (1905-1967), Oberarzt der Heilanstalt Hohenlychen, im KZ Neuengamme [27] und die Experimente von Gualtherus Zahn (Lebensdaten unbekannt) im KZ Sachsenhausen. Nicht immer litten die Opfer schon vor Beginn der Experimente an Tbc, sondern wurden, wie bei den Versuchen von Heißmeyer in Neuengamme, zunächst künstlich mit Tuberkelbazillen infiziert. Heißmeyers Versuche sind ein besonders erschreckendes Beispiel, weil er dazu neben russischen und serbischen Kriegsgefangenen auch zwanzig jüdische Kinder im Alter von 5 - 12 Jahren heranzog, die nach dem Ende der Experimente am 20. April 1945 zur Beseitigung von Spuren ermordet wurden. Bei den Versuchen ging es um die Frage, ob sich der Zustand von Lungentuberkulösen durch gezieltes Setzen eines weiteren Tuberkuloseherds auf der Haut günstig beeinflussen ließ. Um die Wirkung des Verfahrens zu überprüfen, entnahm Heißmeyer den Kindern vor ihrer Ermordung die Lymphknoten und schickte sie zur histologischen Untersuchung ins Berliner Rudolf-Virchow-Krankenhaus [28].

Nicht alle Experimente lassen sich heute noch exakt rekonstruieren, weil die damals geführten Versuchsprotokolle zum Teil nicht mehr auffindbar sind. Ein Beispiel sind die Tbc-Versuche des niederländische Mediziners Gualtherus Zahn im KZ Sachsenhausen. Diese können aber - zumindest in Grundzügen - anhand von Zeugenaussagen und Erinnerungsberichten nachgezeichnet werden. So gab der leitende SS-Lagerarzt Heinz Baumkötter (1912 - 2001) im Jahr 1946 an, dass in Sachsenhausen „eine experimentelle Station zur Heilung der Tuberkulose“ existierte. In dieser habe „der holländische Arzt Za[h]n“ eine „Tuberkuloseheilmethode mittels Inhalation“ erprobt, allerdings hätten seine Experimente „keine positiven Resultate“ ergeben und seien deshalb Ende 1943 abgebrochen worden [29]. Der Beginn der Versuche wurde von dem zu jener Zeit als Pfleger in der Tbc-Abteilung tätigen Häftling Wilhelm Thierhoff (1903-1993) auf 1940 datiert [30]. Weitere Informationen zu den Versuchen enthält ein Erinnerungsbericht des ehemaligen niederländischen Häftlings J.E.A. Post Uiterweer (geb. 1917): „Neben der Baracke, wo ich arbeitete, stand eine ganz kleine Baracke, wo zwei Therapien für Tbc-Erkrankte ausprobiert wurden. [...] In jedem Zimmer waren immer in den einander gegenüberliegenden Betten Häftlinge mit diesen Tbc-Kavernen (Löcher in der Lunge) gelegen. Beide bekamen sie verschiedene Therapien. Der eine sollte täglich mehrmals Säure inhalieren. Dafür war ein Apparat an der Wand angebracht. Dem anderen wurden edle Metalle injiziert. [...] Wie ich es verstanden habe, war es Sache herauszukriegen, welche Therapie die beste war ..." [31] Uiterweers Bericht verdeutlicht, dass Zahn - anders als Heißmeyer in Neuengamme - bereits an Tbc erkrankte Häftlinge zu seinen Experimenten heranzog. Bei den Versuchen verabreichte er einem Teil von ihnen ein - möglicherweise von ihm selbst entwickeltes - Inhalat. Die Vergleichsgruppe erhielt Injektionen mit einer Substanz, bei der es sich aber vermutlich um ein Plazebo handelte. Leider machten weder Uiterweer noch andere Zeugen Angaben zur Zahl der Versuchspersonen und auch die Beschaffenheit des erprobten Inhalats bleibt weitgehend im Dunkeln. Nicht mehr zu klären ist schließlich auch die brisante Frage, weshalb der Niederländer Zahn - den SS-Arzt Baumkötter als „außerhalb des Lagers“ wohnenden „Zivilarzt" beschrieb [32] - im KZ Sachsenhausen an Häftlingen experimentierte.

Wie der Beitrag zeigen soll, wurden Tuberkulöse im Nationalsozialismus gesellschaftlich benachteiligt, planmäßig vernachlässigt und in Konzentrationslagern sogar gezielt getötet oder als Versuchspersonen für medizinische Experimente missbraucht. Ideologischer Hintergrund dieser Repression war die Wahrnehmung der Tuberkulose als „asoziale Krankheit“. Die von den Nationalsozialisten eingeführten Maßnahmen gegen Tbc zielten hauptsächlich darauf ab, die (erb-) gesunde Bevölkerung vor der Krankheit zu schützen. Die noch während der Weimarer Republik praktizierte wirtschaftliche und medizinische Fürsorge für die Erkrankten wurde dagegen stark reduziert. Dieser radikale Umschwung nach 1933 lässt ermessen, wie stark ein politischer Wandel den gesellschaftlichen Umgang mit einer Krankheit zu beeinflussen vermag. 
${ }^{17} \mathrm{Zu}$ den Aufgaben der Gesundheitsämter in der „Tuberkulosefürsorge“ im einzelnen: Klein (Anm. 6).

${ }^{1}$ Otto P. Hornstein, Tb ist auch eine politische Seuche, in: Nordbayerische Zeitung vom 24.3.2005, S. 7.

2 Max Mosse und Gustav Tugendreich, Krankheit und soziale Lage, München 1913.

${ }^{3}$ Die Ausführungen zur kollektiven Wahrnehmung der Tuberkulose folgen der Darstellung von Sylvelyn Hähner-Rombach, Sozialgeschichte der Tuberkulose. Vom Kaiserreich bis zum Ende des Zweiten Weltkriegs unter besonderer Berücksichtigung Württembergs, Stuttgart 2000, S. 29-35.

4 Geprägt wurde der Begriff „Proletarierkrankheit“ allerdings erst im Jahre 1912 durch den Sozialhygieniker und späteren Begründer der Arbeitsmedizin in Bayern, Franz Koelsch (1876 - 1970): Koelsch zufolge hatte die Krankheit in der Arbeiterschaft „einen so erschreckenden Umfang an[genommen]“, dass ihm „die Bezeichnung 'Proletarierkrankheit' gerechtfertigt" schien: Franz Koelsch, Tuberkulose und Beruf, in: Handwörterbuch der sozialen Hygiene, hgg. von Alfred Grotjahn und J. Kaup, Bd. 2, Leipzig 1912, S. 650-655, hier S. 650.

${ }^{5}$ Zum Konzept der „Leistungsmedizin“: Karl-Peter Reeg, Friedrich Georg Christien Bartels (1892-1968). Ein Beitrag zur Entwicklung der Leistungsmedizin im Nationalsozialismus, Husum 1988.

${ }^{6}$ Franz Klein, Die Aufgaben der Tuberkulosefürsorge und die hierbei erforderliche Zusammenarbeit der Gesundheitsämter mit den praktischen Aerzten, Heilstätten und Parteistellen, in: Veröffentlichungen aus dem Gebiet Volksgesundheitsdienstes 50 (1938), S. 595-622, hier S. 602.

${ }^{7}$ Richard Bochalli, Die Geschichte der Schwindsucht, Leipzig 1940; Jürgen Voigt, Tuberkulose. Geschichte einer Krankheit, Köln 1994.

8 Alfred Grotjahn, Soziale Pathologie, Berlin 1912, S. 91.

${ }^{9}$ Karl Diehl und Otmar von Verschuer, Zwillingstuberkulose, Jena 1933; dies., Der Erbeinfluß bei der Tuberkulose. Zwillingstuberkulose II, Jena 1936.

10 Karl Diehl, Erkrankungen der Atemorgane und die Tuberkulose unter dem Gesichtspunkt der Vererblichkeit, in: Wer ist erbgesund und wer ist erbkrank? Praktische Ratschläge für die Durchführung des Gesetzes „zur Verhütung erbkranken Nachwuchses“ [...], hgg. von Wilhelm Klein, Jena 1935, S. 56-88, hier S. 87.

11 Siehe: Kerstin Kelting, Das Tuberkuloseproblem im Nationalsozialismus, Diss. med. Kiel 1974.

${ }^{12} \mathrm{Zu}$ Inhalt und Auswirkungen des NS-Sterilisationsgesetzes: Astrid Ley, Zwangssterilisation und Ärzteschaft. Hintergründe und Ziele ärztlichen Handelns 1934-1945, Frankfurt/M. 2004, bes. S. 34-120.

13 „Gesetz zur Verminderung der Arbeitslosigkeit“ vom 1.6.1933, Abschnitt V, in: Reichsgesetzblatt I, 1933, S.377; „Zweite Durchführungsverordnung des Reichsministers der Finanzen über die Gewährung von Ehestandsdarlehen“ vom 26.7.1933, in: ebd., S. 540. Vgl. hierzu auch: Hähner-Rombach (Anm. 3), S. 264 f.

14 „Gesetz zum Schutze der Erbgesundheit des deutschen Volkes“ vom 18.10.1935, in: Reichsgesetzblatt I, 1935, S. 1246; Arthur Gütt, Herbert Linden und Franz Maßfeller, Blutschutz und Ehegesundheitsgesetz, München 1937. Siehe auch: Hähner-Rombach (Anm. 3), S. 265 f.

15 Ebd., S $266 \mathrm{f}$.

16 „Verordnung zur Bekämpfung übertragbarer Krankheiten“ vom 1.12.1938, in: Reichsgesetzblatt I, 1938, S. 1721 - 1724. Mit dieser Verordnung vereinheitlichte die NS-Regierung die auf Länderebene bestehenden Regelungen aus der Zeit vor und nach 1933. Die damit reichsweit statuierte allgemeine Meldepflicht bei Tuberkulose war zwar schon seit dem Ende des 19. Jahrhunderts diskutiert worden, sie hatte sich aber - nicht zuletzt wegen des Widerstands frei praktizierender Ärzte - bisher politisch nicht durchsetzen lassen. Zur Diskussion um die Meldepflicht siehe: Flurin Condrau, Lungenheilanstalt und Patientenschicksal. Sozialgeschichte der Tuberkulose in Deutschland und England im späten 19. und frühen 20. Jahrhundert, Göttingen 2000, S. 83 f. Zur Tuberkulosegesetzgebung 1871-1975 sowie zum ärztlichen Widerstand gegen die Meldepflicht: Hähner-Rombach (Anm. 3), S. 240-243, $271 \mathrm{f}$.

18 Ebd., S. $601 \mathrm{f}$

${ }^{19}$ Hähner-Rombach (Anm 3), S. 272 - 274.

${ }^{20}$ A. Aschenbrenner, Die Wirkung der Zwangsasylierung auf asoziale Offentuberkulöse, in: Beiträge zur Klinik der Tuberkulose 94 (1940), S.635-641, hier S.637; Gerhard Kloos, Die Durchführung der Zwangsunterbringung von rücksichtslosen Offentuberkulösen, in: Deutsches Tuberkulose-Blatt 16 (1942), S. 222-229, 242-246, hier S. 228. Zum Vorgehen in Stadtroda siehe auch: Hähner-Rombach (Anm. 3), S. 275-279.

21 Günther Wackernagel, Zur Errichtung und Funktion des Häftlingsreviers von 1937 bis 1940, S. 3 (Manuskript: Archiv der Gedenkstätte Sachsenhausen [AS], P3 Wackernagel).

22 Zeugenaussage von Leo Clasen im Strafverfahren gegen Heinz Baumkötter u.a. vor dem Landgericht Münster 1961/62 (Kopie: AS, JD 1/11, S. 82-87).

${ }^{23}$ Vgl.: Protokoll von Berichten und Diskussionen beim Workshop „Der Umgang mit geschwächten Häftlingen in Konzentrationslagern [...]“, 20. -22.6.2004, Gedenkstätte Buchenwald (Kopie: AS); Emilio Büge, 1047 KZ-Geheimnisse (Manuskript: AS, LAG I/3).

${ }^{24}$ Zur Problematik medizinischer Experimente an KZ-Häftlingen im Allgemeinen: Gerhard Baader, Menschenversuche in Konzentrationslagern, in: Johanna Bleker und Norbert Jachertz (Hg.), Medizin im Dritten Reich, Köln 1989, S. 103 - 111; Astrid Ley und Marion Maria Ruisinger (Hg.): Gewissenlos - gewissenhaft. Menschenversuche im Konzentrationslager. Katalog zur Ausstellung des Instituts für Geschichte der Medizin, Universität Erlangen-Nürnberg, Erlangen 2001.

${ }^{25}$ Ernst Klee, Auschwitz, die Medizin und ihre Opfer, Frankfurt/M. 1997, S. $40 \mathrm{f}$.

${ }^{26}$ Christine Wolters, „Zur Belohnung wurde ich der Malaria-Versuchsstation zugeteilt ..." Die Karriere des Dr. Rudolf Brachtel, in: Ralph Gabriel u.a. (Hg.), Lagersystem und Repräsentation. Interdisziplinäre Studien zur Geschichte der Konzentrationslager, Tübingen 2004 , S. 29- 45 .

27 Günther Schwarberg, Der SS-Arzt und die Kinder vom Bullenhuser Damm, Göttingen 1988; Paul Weindling, Genetik und Menschenversuche 1940 - 1950. Hans Nachtsheim, die Kaninchen von Dahlem und die Kinder vom Bullenhuser Damm, in: Hans-Walter Schmuhl (Hg.), Rassenforschung an den Kaiser-Wilhelm-Instituten vor und nach 1933, Göttingen 2003, S. 245-274; Herbert Diercks, Gesucht wird: Dr. Kurt Heißmeyer, in: Beiträge zur Geschichte der nationalsozialistischen Verfolgung in Norddeutschland, Heft 9, Hamburg 2005, S. $102-115$.

${ }^{28}$ Wie Paul Weindling kürzlich herausgearbeitet hat, ging es bei den Versuchen offenbar nicht nur ein therapeutisches Verfahren sondern auch um Fragen aus den Bereichen „Rassenforschung“ und Erbpathologie: So war Heißmeyer an einem möglichen Zusammenhang von Rassenzugehörigkeit und Tbc-Anfälligkeit interessiert, der beteiligte Pathologe aus dem Virchow-Krankenhaus forschte zur sogenannten Pelger-Anomalie (heute: Pelger-Huet-Kernanomalie), die im Verdacht stand, die Anfälligkeit für Tuberkulose zu erhöhen. Siehe: Weindling (Anm 27)

29 Zeugenaussage von Heinz Baumkötter im Strafverfahren gegen Anton Kaindl u. a. vor dem Militärtribunal der Gruppe der Sowjetischen Besatzungstruppen in Deutschland 1946/47 (Kopie: AS, JSU 1/21, S. $14-17$, hier S. 14).

30 Zeugenaussage von Wilhelm Thierhoff im Ermittlungsverfahren gegen Ludwig Ehrsam vor dem Landgericht Köln 1968 (Kopie: AS, JD 8/2, Teil 2, S. 170-180).

31 J.E.A Post Uiterweer, Versuchsanstalt für Tbc im KZ Sachsenhausen, 1990, S.1 f. (Kopie: AS, P3 Thierhoff). Grammatikalische Fehler in dem Bericht des Niederländers wurden von der Verfasserin korrigiert.

32 Zeugenaussage Baumkötter (Anm. 29). 